\title{
Turbulent fluctuations around Bjorken flow
}

\author{
Stefan Floerchinger and Urs Achim Wiedemann \\ Physics Department, Theory Unit, CERN, CH-1211 Genève 23, Switzerland
}

\begin{abstract}
We study the evolution of local event-by-event deviations from smooth average fluid dynamic fields, as they can arise in heavy ion collisions from the propagation of fluctuating initial conditions. Local fluctuations around Bjorken flow are found to be governed by non-linear equations whose solutions can be characterized qualitatively in terms of Reynolds numbers. Perturbations at different rapidities decouple quickly, and satisfy (after suitable coordinate transformations) an effectively two-dimensional Navier-Stokes equation of non-relativistic form. We discuss the conditions under which non-linearities in these equations cannot be neglected and turbulent behavior is expected to set in.
\end{abstract}

In recent years, hadronic transverse momentum spectra and their azimuthal dependence with respect to the orientation of the reaction plane have provided tight constraints on the fluid dynamic model of ultra-relativistic heavy ion collisions. With the first data from the LHC, and with refined analyses of RHIC data, higher order flow coefficients $v_{3}, v_{4}, v_{5}$ and $v_{6}$ are now starting to complement the measurements of elliptic flow $v_{2}$. This provides access to qualitatively novel features of the collision dynamics. In particular, since event-averaged initial conditions of heavy ion collisions are by construction symmetric with respect to the reaction plane at mid rapidity, the recent measurements of non-vanishing odd harmonic coefficients $v_{3}, v_{5}$ provide unambiguous evidence for the relevance of event-by-event fluctuations in the fluid dynamic evolution. Remarkably, at least some of the models currently used to specify initial conditions of fluid dynamic simulations can provide naturally for initial event-by-event fluctuations of the phenomenologically required size [1]. First studies of the fluid dynamic propagation of such geometric initial state fluctuations have resulted in marked improvements in the comparison of fluid dynamic simulations with data [2].

Motivated by these developments, we are studying here the evolution of local eventby-event deviations from smooth average fluid dynamic fields within the expanding geometry characteristic for ultra-relativistic heavy ion collisions. We are for instance interested in how a 'primordial' spectrum of such fluctuations evolves, and which modes of the fluctuation spectrum are damped on which time scales. Given that fluid dynamic fluctuations measure deviations from equilibrium, we hope to gain in this way novel access to the basic problem of how equilibration can proceed efficiently in heavy collisions. We also wonder to what extent it is justified to propagate primordial fluctuations in a linearized ansatz that by the nature of its approximation leaves no room for the development of turbulent phenomena. Such an approach is well-motivated 
in the treatment of primordial fluctuations in cosmology, but as we shall discuss in the following, the scales and flows in heavy ion collisions can support a qualitatively different conclusion.

To be specific, we consider local fluctuations in the fluid dynamic velocity $\delta u^{\mu}$ and energy density $\delta \epsilon$ on top of average fields $\bar{u}^{\mu}$ and $\bar{\epsilon}$ that satisfy Bjorken's scaling solution. We work in light cone coordinates, where $\bar{u}^{\mu}=(1,0,0,0)$ and $\bar{\epsilon}=\epsilon_{B j}\left(\tau_{0}\right)\left(\tau_{0} / \tau\right)^{4 / 3}$. In general, one can split at fixed time $\tau$ an arbitrary velocity field $u^{\mu}=\bar{u}^{\mu}+\delta u^{\mu}$ into an irrotational part represented by the divergence

$$
\vartheta=\partial_{1} u^{1}+\partial_{2} u^{2}+\partial_{y} u^{y}
$$

and a solenoidal part represented by the vorticity field

$$
\omega_{1}=\tau \partial_{2} u^{y}-\frac{1}{\tau} \partial_{y} u^{2}, \quad \omega_{2}=\frac{1}{\tau} \partial_{y} u^{1}-\tau \partial_{1} u^{y}, \quad \omega_{3}=\partial_{1} u^{2}-\partial_{2} u^{1} .
$$

Because of the normalization $u^{\mu} u_{\mu}=-1$, there are only three independent components $\delta u^{j}$ which we choose to span the transverse plane and rapidity, $j=1,2, y$. The energy density $\epsilon=\bar{\epsilon}+\delta \epsilon$ can be characterized in terms of temperature deviations from its Bjorken value

$$
\hat{d}=\ln \left(T / T_{\mathrm{Bj}}(\tau)\right)
$$

Within this set-up, we have derived evolution equations for $\delta u^{j}$ and $\delta \epsilon$ from the relativistic viscous fluid dynamic equations for $u^{\mu}$ and $\epsilon$.

We first comment on the linearized evolution equations for $\delta u^{j}, \delta \epsilon$. In this case, the equations for $\vartheta$ and $\hat{d}$ are coupled and describe essentially the propagation of sound (modulo modifications due to the expanding background, see Ref. [4]). The vorticity modes satisfy a diffusion-type equation of motion that can be solved directly. In Fourrier space, denoting by $k_{y}$ the wave number conjugate to rapidity,

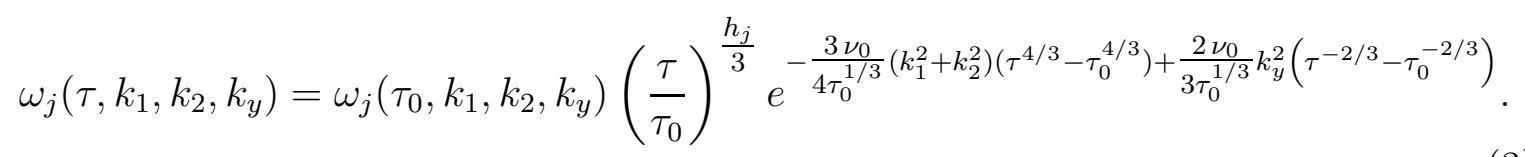

For this solution, we assumed a $\tau$-independent ratio $\eta / s$ that leads to a kinematic viscosity $\nu_{0}=\eta /(\epsilon+p)=\eta /(s T)$ evaluated at time $\tau_{0}$. While the transverse vorticity components $\left(h_{1}=h_{2}=-2\right)$ fall off like $1 / \tau^{2 / 3}$ for small wave-vectors or small kinematic viscosity $\nu_{0}$, the vorticity mode $\omega_{3}$ grows algebraically in this region $\left(h_{3}=1\right)$. Therefore, at least in a linearized description, there can be initial fluctuations that do not attenuate within the phenomenologically relevant time scales of heavy ion collisions, see Fig. 1.

In general, a linearized formalism applies if perturbations are small $\delta \epsilon / \bar{\epsilon} \ll 1, d \ll 1$ and if Reynolds numbers are small,

$$
\operatorname{Re}=\frac{u_{T} l(\epsilon+p)}{\eta}=\frac{u_{T} l s T}{\eta} .
$$

Here $u_{T}$ is a characteristic velocity of fluctuations in the transverse direction and $l$ is the typical length scale over which it changes significantly. (For motion in the 


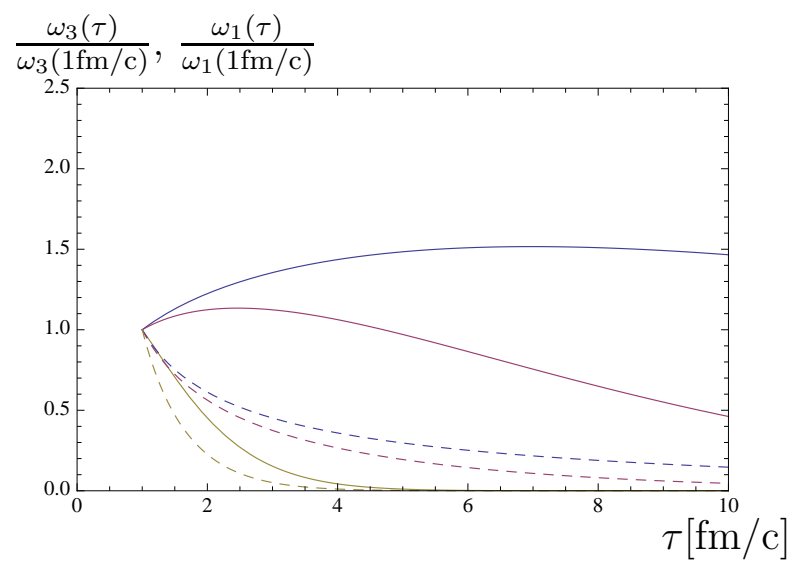

Figure 1. Vorticity amplitudes $\omega_{3}(\tau) / \omega_{3}(1 \mathrm{fm} / \mathrm{c})$ (solid lines) and $\omega_{1}(\tau) / \omega_{1}(1 \mathrm{fm} / \mathrm{c}$ ) (dashed lines) for wave vectors with $k_{2}=k_{y}=0$, and $k_{1}=5 \mathrm{fm}^{-1}, k_{1}=10 \mathrm{fm}^{-1}$ and $k_{1}=30 \mathrm{fm}^{-1}$, respectively. Viscosity has been chosen as $\nu(\tau) / \tau=10^{-3}$ at $\tau=1 \mathrm{fm} / \mathrm{c}$.

rapidity direction, there is a different Reynolds number [4].) If the Reynolds number is large, then turbulent flow sets in and a linearized formalism fails. In this case, the smallness of perturbations around an expanding background still leads to the important simplification that the fluid can be treated as compression-less, $\vartheta=0$. This condition does not mean that there are no sound waves present, but that the coupling between sound and turbulence becomes negligible. The formal criterion is a small Mach number

$$
\mathrm{Ma}=\frac{\sqrt{u_{1} u^{1}+u_{2} u^{2}+u_{y} u^{y}}}{c_{S}} \ll 1,
$$

where $c_{S}$ is the velocity of sound. It is conceivable that the condition Ma $\ll 1$ for compression-less turbulent flow is realized in heavy ion collisions.

In terms of a rescaled time $t=3 \tau^{4 / 3} /\left(4 \tau_{0}^{1 / 3}\right)$, temperature field $d=\left(\tau_{0} / \tau\right)^{2 / 3} \hat{d}$ and velocity $v_{j}=\left(\tau_{0} / \tau\right)^{1 / 3} u_{j}$, we find that the non-linear fluid equations $(j=1,2, y)$ take the form

$$
\partial_{t} v_{j}+\sum_{m=1}^{2} v_{m} \partial_{m} v_{j}+\frac{1}{\tau^{2}} v_{y} \partial_{y} v_{j}+\partial_{j} d-\nu_{0}\left(\partial_{1}^{2}+\partial_{2}^{2}+\frac{1}{\tau^{2}} \partial_{y}^{2}\right) v_{j}=0
$$

with the solenoidal constraint $\partial_{1} v_{1}+\partial_{2} v_{2}+\frac{1}{\tau^{2}} \partial_{y} v_{y}=0$. For late times, this becomes effectively a two-dimensional Navier-Stokes equation of non-relativistic form for a twodimensional compression-less fluid. Therefore, the development of turbulent flow in heavy ion collisions can be discussed on the basis of an equation about which much is known already. In particular, for the three-dimensional Navier-Stokes equation, one knows that kinetic energy cascades in the case of turbulence from large structures in space to finer and finer ones where it is eventually dissipated. In contrast, in two dimensions, turbulent kinetic energy is subject to an inverse cascade from microscopic to more and more macroscopic structures. This plays a role for turbulent phenomena in the essentially two-dimensional layer of the Earth's atmosphere and it prompts us 


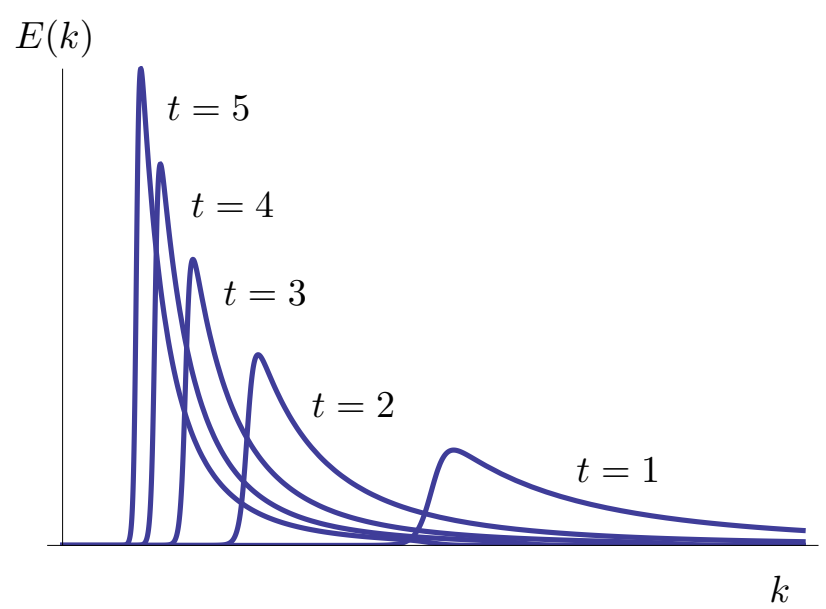

Figure 2. Illustration of turbulent kinetic energy as a function of the wave-number for freely decaying turbulence according to Batchelors scaling theory.

to wonder whether (6) allows for a scale amplification mechanism of some fluctuating modes in heavy ion collisions.

One way to characterize the inverse cascade is to study how the kinetic energy in a fluid,

$$
\lambda^{2}=\frac{1}{2}\left\langle v_{1}^{2}+v_{2}^{2}\right\rangle=\int_{0}^{\infty} d k E(k),
$$

is distributed over wave vectors $E(k)$ as a function of time. Based on the theory for turbulence developed by Kolmogorov in three and Kraichnan in two dimensions, there is in particular a scaling theory for freely decaying two-dimensional turbulence that leads to $E(t, k)=\lambda^{3} t h(k \lambda t)$, where $h(x)$ is conjectured to be universal [3]. In this case, kinetic energy accumulates at small wave-vectors at late times, see Fig. 2.

The scaling theories of Kolmogorov, Kraichnan and Batchelor address turbulence at very large Reynolds number. Estimates of Reynolds numbers realized in heavy ion collisions are usually of the order $\operatorname{Re} \approx s / \eta=\mathcal{O}(10)$. This is too small for applying results from fully developed turbulence, but it is sufficiently large to motivate a search for the onset of turbulent phenomena involving fundamental quantum fields in heavy ion collisions. This is a report on work in progress. We are currently exploring possible signatures for the onset of turbulent phenomena in measurements of single inclusive hadron spectra and two-particle correlation functions.

[1] B. Alver and G. Roland, Phys. Rev. C 81 (2010) 054905 [Erratum-ibid. C 82 (2010) 039903].

[2] H. Holopainen, H. Niemi and K. J. Eskola, Phys. Rev. C 83, 034901 (2011)

[3] G. K. Batchelor, Phys. Fluids Suppl. II 12, 223 (1969).

[4] S. Floerchinger and U. A. Wiedemann, work in preparation. 